\title{
Effect of Nerve-Sparing Radical Prostatectomy on Urinary Continence in Patients With Preoperative Erectile Dysfunction
}

\author{
Yong Hyun Park, Oh Seong Kwon, Sung-Hoo Hong, Sae Woong Kim, Tae-Kon Hwang, Ji Youl Lee \\ Department of Urology, Seoul St. Mary's Hospital, The Catholic University of Korea School of Medicine, Seoul, Korea
}

Purpose: We aimed to assess whether nerve-sparing radical prostatectomy (nsRP) is associated with improved recovery of urinary continence compared to non-nerve-sparing radical prostatectomy (nnsRP) in patients with localized prostate cancer and preoperative erectile dysfunction.

Methods: A total of 360 patients with organ-confined prostate cancer and an International Index of Erectile Function score of less than 17 were treated with nsRP or nnsRP in Seoul St. Mary's Hospital. Patients who received neoadjuvant or adjuvant androgen deprivation therapy or had a history of prostate-related surgery were excluded. Recovery of urinary continence was assessed at $0,1,3,6$, and 12 months. Postoperative recovery of continence was defined as zero pad usage. The association between nerve-sparing status and urinary continence was assessed by using univariate and multivariate Cox regression analyses after controlling for known predictive factors.

Results: Urinary continence recovered in 279 patients (77.5\%) within the mean follow-up period of 22.5 months (range, 6-123 months). Recovery of urinary continence was reported in $74.6 \%$ and $86.4 \%$ of patients after nnsRP and nsRP, respectively, at 12 months $(\mathrm{P}=0.022)$. All groups had comparable perioperative criteria and had no significant preoperative morbidities. Age, American Society of Anesthesiologists score, and nerve-sparing status were significantly associated with recovery of urinary continence on univariate analysis. On multivariate analysis, age (hazard ratio [HR], 1.254; 95\% confidence interval $[\mathrm{CI}], 1.002-1.478 ; \mathrm{P}=0.026)$ and nerve-sparing status ( $\mathrm{HR}, 0.713 ; 95 \% \mathrm{CI}, 0.548-0.929 ; \mathrm{P}=0.012)$ were independently associated with recovery of urinary continence.

Conclusions: nsRP, as compared to nnsRP, improves recovery rates of urinary incontinence and decreases surgical morbidity without compromising pathologic outcomes.

Keywords: Prostate Neoplasms; Prostatectomy; Nerve-Sparing; Urinary Incontinence; Erectile Dysfunction

- Grant Support: This study was supported by a grant from the Korean Health Technology R\&D Project, Ministry of Health \& Welfare, Republic of Korea (HI14C1203).

- Research Ethics: After approval from the Institutional Review Board at the Catholic University of Korea, Seoul St. Mary's Hospital (approval number: KC14RISI0934), clinical data of eligible patients were extracted from the Smart Prostate Cancer database of Seoul St. Mary's Hospital.

- Conflict of Interest: No potential conflict of interest relevant to this article was reported.

\section{INTRODUCTION}

Prostate cancer is one of the most common cancers worldwide. In Korea, the incidence of prostate cancer increased from 8.4 per
100,000 population in 1999 to 27.4 per 100,000 in 2011. This increase is the second highest among cancers affecting men. Conversely, 5-year relative survival rates have improved from 55.9\% in patients diagnosed from 1993 to 1995 to $92.0 \%$ in those diag-
Corresponding author: Ji Youl Lee (iD http://orcid.org/0000-0001-6775-1157 Department of Urology, Seoul St. Mary's Hospital, The Catholic University of Korea School of Medicine, 222 Banpo-daero, Seocho-gu, Seoul 06591, Korea E-mail: uroljy@catholic.ac.kr / Tel: +82-2-2258-6227 / Fax: +82-2-599-7839 Submitted: August 28, 2015 / Accepted after revision: October 18, 2015
C) $\$$ This is an Open Access article distributed under the terms of the Creative Commons Attribution Non-Commercial License (http://creativecommons.org/licenses/by-nc/4.0/) which permits unrestricted non-commercial use, distribution, and reproduction in any medium, provided the original work is properly cited. 
nosed from 2007 to 2011 [1]. With increasing survival rates, quality of life issues have become important concerns for patients undergoing radical treatment of prostate cancer, as well as for their physicians. Radical prostatectomy is therefore assessed based on functional outcomes, which include urinary incontinence and erectile dysfunction, in addition to oncologic outcomes.

Several surgical techniques have been described for achieving recovery of urinary continence after radical prostatectomy [2-4]. However, a recent meta-analysis has identified posterior reconstruction with or without anterior reconstruction as the only procedure that enabled significant early recovery of urinary continence [5]. The role of nerve-sparing approach in enabling early recovery of urinary continence remains controversial [6-9]. Inclusion of patient groups with variable degrees of preoperative erectile function could have possibly contributed to the limitations of prior studies in addressing this controversy. Therefore, this study was designed to assess whether nervesparing radical prostatectomy (nsRP) enabled recovery of urinary continence as compared to non-nerve-sparing radical prostatectomy (nnsRP) in patients with localized prostate cancer and preoperative erectile dysfunction.

\section{MATERIALS AND METHODS}

A total of 360 patients who underwent radical prostatectomy between January 2003 and December 2012 and meeting the following criteria were included: (1) organ-confined prostate cancer, (2) preoperative erectile dysfunction (International Index of Erectile Function-5 [IIEF-5] score < 17), (3) no neoadjuvant or adjuvant androgen deprivation therapy or radiation therapy, (4) no previous prostate-related surgery, (5) a follow-up duration of over 1 year. After approval from the Institutional Review Board at the Catholic University of Korea, Seoul St. Mary's Hospital (approval number: KC14RISI0934), clinical data of eligible patients were extracted from the Smart Prostate Cancer database of Seoul St. Mary's Hospital [10]. Extracted data included age, body mass index, American Society of Anesthesiologists (ASA) score, preoperative prostate-specific antigen (PSA) level, clinical stage, biopsy Gleason score, and pathologic outcomes. Preoperative lower urinary tract symptoms and erectile function were assessed before surgery by using the International Prostate Symptom Score (IPSS) and IIEF-5 questionnaires, respectively.

Radical prostatectomy was performed by using a laparoscopic $(n=275,76.4 \%)$ or robotic $(n=85,23.6 \%)$ approach. Nervesparing prostatectomy was performed by using the interfascial technique described by Gaston and colleagues [11], and was selected by each physician, independent of preoperative erectile function. Recovery of urinary continence was assessed at 1, 3, 6, and 12 months postoperatively and every 3-6 months thereafter. Postoperative recovery of urinary continence was defined as zero pad usage.

IBM SPSS Statistics ver. 19.0 (IBM Co., Armonk, NY, USA) was used for analysis. Data was compared by using MannWhitney U-test for continuous variables and chi-square test for categorical variables. Kaplan-Meier analysis assessed time to recovery of urinary continence according to nerve-sparing status. The association between nerve-sparing status and urinary continence was assessed by using univariate and multivariate Cox regression analyses, after controlling for known predictive factors.

\section{RESULTS}

All baseline parameters, except preoperative IIEF-5 score and preoperative serum PSA level, were comparable between the two groups (Table 1). The mean operative times for the nsRP and nnsRP groups were 199.7 and 206.5 minutes, respectively $(\mathrm{P}=0.044)$. The mean estimated blood loss was $391.1 \mathrm{~mL}$ in the nsRP group and $415.3 \mathrm{~mL}$ in the nnsRP group respectively $(\mathrm{P}=0.489)$ (Table 2$)$. The nnsRP group had a significantly higher perioperative complication rate than the nsRP group $(2.0 \%$ vs. $17.0 \%, \mathrm{P}=0.003$ ). However, complications were mostly less than grade III. Table 2 shows the pathologic outcomes of both the nsRP and nnsRP groups. There were no significant differences in pathologic T stage, pathologic Gleason score, and positive surgical margins between the nsRP and nnsRP groups.

At the mean follow-up of 22.5 months (range, 6-123 months), urinary continence recovery was noted in 279 patients (77.5\%). Urinary continence recovery rates at 3, 6, and 12 months were $33.8 \%, 58.3 \%$, and $77.6 \%$ in the nnsRP group and $54.5 \%, 72.7 \%$, and $85.2 \%$ in the nsRP group, respectively (Fig. 1, log-rank test, $\mathrm{P}=0.003)$. When patients were stratified according to age $(\leq 70$ yr vs. $>70 \mathrm{yr}$ ), the benefits of nsRP in enabling better urinary continence recovery rates reached statistical significance in older patients (nnsRP vs. nsRP; $69.0 \%$ vs. $95.0 \%, \mathrm{P}=0.014$ ), but not in younger patients $(79.5 \%$ vs. $83.8 \%, \mathrm{P}=0.576)$.

Table 3 shows univariate and multivariate Cox regression analyses for predicting recovery of urinary continence. On univariate analysis, age $>70$ years, ASA score, total IPSS score, and nerve-sparing status were significantly associated with recovery of urinary continence. On multivariate analysis, age $>70$ years 
Table 1. Patient demographics and baseline data

\begin{tabular}{|c|c|c|c|c|}
\hline \multirow{2}{*}{ Variable } & \multirow{2}{*}{ Overall } & \multicolumn{3}{|c|}{ According to nerve-sparing } \\
\hline & & $\operatorname{nsRP}(\mathrm{n}=88)$ & $\operatorname{nnsRP}(\mathrm{n}=272)$ & $\mathrm{P}$-value \\
\hline Age (yr) & $67.3 \pm 6.0$ & $67.1 \pm 6.1$ & $67.6 \pm 6.3$ & 0.482 \\
\hline Body mass index $\left(\mathrm{kg} / \mathrm{m}^{2}\right)$ & $25.5 \pm 2.4$ & $25.3 \pm 2.6$ & $25.5 \pm 2.4$ & 0.532 \\
\hline ASA score & & & & 0.583 \\
\hline 1 & $102(28.7)$ & $23(26.1)$ & $79(29.5)$ & \\
\hline 2 & $243(68.3)$ & $61(69.3)$ & $182(67.9)$ & \\
\hline 3 & $11(3.1)$ & $4(4.5)$ & $7(2.6)$ & \\
\hline \multicolumn{5}{|l|}{ IPSS questionnaire } \\
\hline Total score & $12.0 \pm 7.2$ & $11.0 \pm 7.2$ & $12.3 \pm 7.2$ & 0.198 \\
\hline Quality of life score & $2.5 \pm 1.6$ & $2.3 \pm 1.7$ & $2.6 \pm 1.6$ & 0.103 \\
\hline IIEF-5 score & $8.6 \pm 5.7$ & $10.7 \pm 5.3$ & $8.0 \pm 5.7$ & $<0.001$ \\
\hline Preoperative PSA (ng/mL) & $8.2 \pm 5.2$ & $7.1 \pm 4.6$ & $8.6 \pm 5.8$ & 0.032 \\
\hline Total biopsy cores (n) & $11.9 \pm 0.7$ & $11.8 \pm 0.6$ & $11.9 \pm 0.8$ & 0.759 \\
\hline Positive cores (n) & $2.6 \pm 1.9$ & $2.3 \pm 1.6$ & $2.8 \pm 2.1$ & 0.228 \\
\hline Maximum percent positive cores & $36.7 \pm 23.9$ & $35.0 \pm 23.4$ & $37.3 \pm 24.3$ & 0.515 \\
\hline Clinical stage (\%) & & & & 0.219 \\
\hline $\mathrm{T} 1$ & $104(28.9)$ & $27(30.7)$ & $77(28.3)$ & \\
\hline $\mathrm{T} 2$ & $240(66.7)$ & $60(68.2)$ & $180(66.2)$ & \\
\hline $\mathrm{T} 3$ & $16(4.4)$ & $1(1.1)$ & $15(5.5)$ & \\
\hline Biopsy Gleason score (\%) & & & & 0.073 \\
\hline$\leq 6$ & $191(53.1)$ & $50(56.8)$ & $141(51.8)$ & \\
\hline $7(3+4)$ & $90(25.0)$ & $20(22.7)$ & $70(25.7)$ & \\
\hline $7(4+3)$ & $45(12.5)$ & $15(17.0)$ & $30(11.0)$ & \\
\hline$\geq 8$ & $34(9.4)$ & $3(3.4)$ & $31(11.4)$ & \\
\hline
\end{tabular}

Values are presented as mean \pm standard deviation or number (\%).

nsRP, nerve-sparing radical prostatectomy; nnsRP, non-nerve-sparing radical prostatectomy; ASA, American Society of Anesthesiologists; IPSS, International Prostate Symptom Score; IIEF-5, International Index of Erectile Function-5; PSA, prostate-specific antigen.

Table 2. Perioperative and pathologic outcomes

\begin{tabular}{|c|c|c|c|c|}
\hline \multirow{2}{*}{ Variable } & \multirow{2}{*}{ Overall } & \multicolumn{3}{|c|}{ According to nerve-sparing } \\
\hline & & $\operatorname{nsRP}(\mathrm{n}=88)$ & $\operatorname{nnsRP}(\mathrm{n}=272)$ & P-value \\
\hline Operative time (min) & $216 \pm 67.8$ & $199.7 \pm 66.2$ & $206.5 \pm 67.1$ & 0.044 \\
\hline Estimated blood loss (mL) & $409.2 \pm 252.0$ & $391.1 \pm 275.6$ & $415.3 \pm 243.5$ & 0.489 \\
\hline Transfusion & $28(7.8)$ & $7(8.0)$ & $21(7.7)$ & 0.943 \\
\hline Postoperative hospital stay (day) & $6.1 \pm 2.4$ & $6.0 \pm 2.1$ & $6.1 \pm 2.4$ & 0.207 \\
\hline \multicolumn{5}{|l|}{ Perioperative complication $^{\text {a) }}$} \\
\hline Minor ( $\leq$ grade III) & $25(10.0)$ & $0(0)$ & $25(12.5)$ & 0.009 \\
\hline Major ( $\geq$ grade IV) & $10(4.0)$ & $1(2.0)$ & $9(4.5)$ & 0.432 \\
\hline Pathologic stage (\%) & & & & 0.149 \\
\hline T0 & $2(0.6)$ & $1(1.1)$ & $1(0.4)$ & \\
\hline $\mathrm{T} 2 \mathrm{a}$ & $65(18.1)$ & $22(25.0)$ & $43(15.8)$ & \\
\hline $\mathrm{T} 2 \mathrm{~b}$ & $20(5.6)$ & $6(6.8)$ & $14(5.1)$ & \\
\hline $\mathrm{T} 2 \mathrm{c}$ & $273(75.8)$ & $59(67.0)$ & $214(78.7)$ & \\
\hline Pathologic Gleason score ${ }^{\mathrm{b})}(\%)$ & & & & 0.479 \\
\hline$\leq 6$ & $116(32.6)$ & $33(38.4)$ & $83(30.7)$ & \\
\hline $7(3+4)$ & $130(36.5)$ & $28(32.6)$ & $102(37.8)$ & \\
\hline $7(4+3)$ & $89(25.0)$ & $22(25.6)$ & $67(24.8)$ & \\
\hline$\geq 8$ & $21(5.9)$ & $3(3.5)$ & $18(6.7)$ & \\
\hline Positive surgical margin & $92(25.6)$ & $17(19.3)$ & $75(27.6)$ & 0.123 \\
\hline
\end{tabular}

Values are presented as mean \pm standard deviation or number $(\%)$.

nsRP, nerve-sparing radical prostatectomy; nnsRP, non-nerve-sparing radical prostatectomy.

a) The number of patients was analyzed in each group; overall $=250, \mathrm{nsRP}=50, \mathrm{nnsRP}=200 .{ }^{\mathrm{b}}$ The number of patients was analyzed in each group; overall $=356, \mathrm{nsRP}=86, \mathrm{nnsRP}=270$. 
Table 3. Univariate and multivariate Cox proportional hazard regression analyses for predicting recovery of urinary continence after radical prostatectomy

\begin{tabular}{|c|c|c|c|c|c|c|}
\hline \multirow{2}{*}{ Factor } & \multicolumn{3}{|c|}{ Univariate analysis } & \multicolumn{3}{|c|}{ Multivariate analysis } \\
\hline & HR & $95 \% \mathrm{CI}$ & $\mathrm{P}$-value & HR & $95 \%$ CI & P-value \\
\hline Age $>70 \mathrm{yr}$ & 1.358 & $1.037-1.778$ & 0.014 & 1.254 & $1.002-1.478$ & 0.026 \\
\hline ASA score & 1.329 & $1.025-2.368$ & 0.048 & 1.127 & $0.906-1.361$ & 0.255 \\
\hline IPSS score & 1.020 & $1.002-1.038$ & 0.031 & 0.983 & $0.962-1.127$ & 0.482 \\
\hline Nerve-sparing & 0.649 & $0.410-0.952$ & 0.008 & 0.713 & $0.548-0.929$ & 0.012 \\
\hline
\end{tabular}

HR, hazard ratio; CI, confidence interval; ASA, American Society of Anesthesiologists; IPSS, International Prostate Symptom Score.

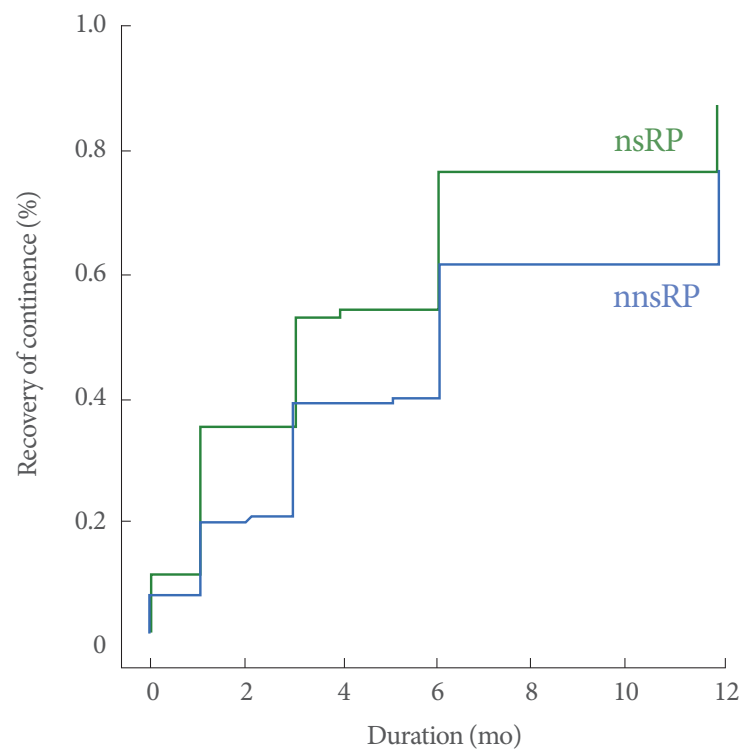

Fig. 1. Urinary continence recovery rates in nsRP and nnsRP $(\mathrm{P}=0.003)$. nsRP, nerve-sparing radical prostatectomy; nnsRP, non-nerve-sparing radical prostatectomy.

(hazard ratio [HR], 1.254; 95\% confidence interval [CI], 1.0021.478; $\mathrm{P}=0.026)$ and nerve-sparing status $(\mathrm{HR}, 0.713 ; 95 \% \mathrm{CI}$, $0.548-0.929 ; \mathrm{P}=0.012$ ) were independently associated with recovery of urinary continence (Table 3 ).

\section{DISCUSSION}

The primary challenge in radical prostatectomy for prostate cancer, as currently recognized, is improvement in the quality of life. Urinary incontinence and erectile dysfunction are among the factors that affect the quality of life in these patients. Although there are several studies on factors predicting early recovery of urinary continence after radical prostatectomy, their results are conflicting about the impact of a nerve-sparing approach on such recovery [6-9,12]. In our study, which compared nnsRP and nsRP in patients with preoperative erectile dysfunction, patients undergoing nsRP demonstrated significantly better rates of recovery of urinary continence. When adjusted for age, the difference between nnsRP and nsRP was statistically significant for older patients ( $>70$ years) alone. Further, in our study, patients treated with nsRP had a $28.7 \%$ reduction in urinary incontinence after adjusting for factors known to be clinically significant for recovery of continence.

Despite the importance of nerve-sparing procedure, many urologists do not choose to perform nsRP in patients with preoperative erectile dysfunction, older age, or advanced disease. Stember et al. [13] defined factors related to nnsRP use as baseline erectile function, biopsy Gleason sum, clinical stage $\geq \mathrm{T} 2$, patient age, and percentage of positive biopsy cores. They demonstrated that patients were one and half (1.5) times more likely to undergo nsRP with every one point increase in the erectile function score. Decisions of patients yield a similar conclusion. Lavery et al. [14] reported that $64 \%$ of patients with moderateto-severe erectile dysfunction prefers nnsRP.

Previous studies have shown significant $[6,7,15-18]$ or no association $[8,9]$ between nsRP and improved recovery of urinary continence. Kundu et al. [8] demonstrated that the recovery of urinary continence was associated with younger age but not with nerve-sparing surgery. Similarly, Tzou et al. [9] reported that attempted or successful nsRP did not result in better rates of urinary continence. However, other studies have reported that attempted or successful nerve-sparing was a statistically significant predictor for recovery of urinary continence when accounting for all other factors by multivariate analysis $[6,7,15]$. A recent systematic review and meta-analysis by Reeves et al. [18] supported the importance of nsRP in enabling early recovery of urinary continence. The meta-analysis demonstrated that nsRP, compared to nnsRP, had significantly better early but not 
long-term continence recovery. Risk ratios were 1.48 (range, 1.34-1.63; $\mathrm{P}<0.001$ ) at $\leq 6$ weeks, 1.24 (range, 1.09-1.42; $\mathrm{P}=0.001$ ) at $3-4$ months, and 1.20 (range, $1.04-1.39 ; \mathrm{P}=0.02$ ) at 6 months, respectively. No significant difference was observed in rates of urinary continence after nsRP and nnsRP at 12 or 24 months. Despite the systematic approach of this review, the significant risk of bias of individual studies diminishes the evidence provided to support the use of nsRP for improved recovery of urinary continence in patients with preoperative erectile dysfunction.

The role of nerve-sparing in patients with preoperative erectile dysfunction is yet to be clarified. Recent studies have demonstrated that nsRP in this population may have advantages beyond improving erectile function $[17,16]$. Khoder et al. [16] treated a total of 420 impotent prostate cancer patients with bilateral intrafascial or interfascial nsRP and found higher continence recovery rates with nerve-sparing procedures. The best results were achieved in the intrafascial nsRP group. Similarly, Harris et al. [17] demonstrated that nerve-sparing improved postoperative urinary but not sexual function in men with low baseline sexual function. All these previous studies suggest that men with preoperative erectile dysfunction may also benefit from nerve-sparing procedures.

Nerve-sparing is important for recovery of urinary continence and this recovery may be based on several proposed mechanisms. First, membranous urethral microcirculation may be a critical part of the continence mechanism. John et al. [19] investigated the results of endoscopic urethral tissue blood flow before and after radical prostatectomy in 37 continent patients. They found that membranous urethral blood flow significantly decreased after radical prostatectomy from 18.8 to $11.9 \mathrm{~mL} /$ $\mathrm{min} / 100 \mathrm{~g}$ of tissue. Patients undergoing nsRP may, therefore, have better pelvic vascular status and more preserved membranous urethral vascular integrity than those undergoing nnsRP. This may contribute to improved recovery of urinary continence after nsRP. Second, there is evidence associating cavernous nerve injury with impaired membranous urethral sensitivity. John et al. [20] found that posterior urethral sensitivity and pressure transmission were impaired immediately after prostatectomy, and improvement in these parameters after 6 months was associated with the restoration of continence. Catarin et al. [21] prospectively identified alterations in the pelvic and membranous urethral afferent and efferent innervation after nsRP by using several neurophysiological tests as well as a validated questionnaire. Sensory and motor pudendal innervation was unaffected after surgery, whereas autonomic afferent denervation of the membranous urethral mucosa was found in $77.3 \%$ of patients. There was significant prevalence of autonomic afferent denervation in patients with occasional leakage of urine (91.7\%).

Improved quality of life and decreased surgical morbidity are critical elements in the decision to choose a nerve-sparing procedure, especially in older patients. This study demonstrates that the benefits of nsRP in terms of improvement in recovery of urinary continence is significant in older ( $>70$ years) rather than younger patients ( $\leq 70$ years). Khoder et al. [16] demonstrated that older patients ( $>70$ years) had a higher rate of full continence with nsRP (80\%) compared to nnsRP (65\%) and this difference tended towards significance $(\mathrm{P}=0.052)$. The reason for this difference based on age may be owing to excellent continence outcomes in the younger age group independent of nerve-sparing procedure. Perioperative complication rates and operative time were significantly lower in the nsRP group than the nnsRP group in our study. A previous study comparing nerve-sparing and wide-excision groups similarly showed a significant difference in operative time (65 and 70 minutes, respectively, $\mathrm{P}<0.001)$ and blood loss $(150 \mathrm{~mL}$ and $200 \mathrm{~mL}$, respectively, $\mathrm{P}<0.001$ ) between the 2 groups [16]. The advantages observed by us should encourage surgeons to perform nsRP more frequently to improve quality of life and minimize surgical morbidity, even in older patients with preoperative erectile dysfunction.

In conclusion, our study involving patients with localized prostate cancer and preoperative erectile dysfunction provides evidence supporting the role of nsRP in enabling improved recovery rates of urinary continence. Additionally, nsRP was found to decrease surgical morbidity when compared to nnsRP, without compromising pathologic outcomes. Based on our study, we recommend surgeons to consider nsRP whenever oncologically and technically feasible irrespective of patients' age or status of erectile dysfunction.

\section{REFERENCES}

1. Jung KW, Won YJ, Kong HJ, Oh CM, Lee DH, Lee JS. Cancer statistics in Korea: incidence, mortality, survival, and prevalence in 2011. Cancer Res Treat 2014;46:109-23.

2. Finley DS, Osann K, Chang A, Santos R, Skarecky D, Ahlering TE. Hypothermic robotic radical prostatectomy: impact on continence. J Endourol 2009;23:1443-50. 
3. Lei Y, Alemozaffar M, Williams SB, Hevelone N, Lipsitz SR, Plaster $\mathrm{BA}$, et al. Athermal division and selective suture ligation of the dorsal vein complex during robot-assisted laparoscopic radical prostatectomy: description of technique and outcomes. Eur Urol 2011;59: 235-43.

4. Joshi N, de Blok W, van Muilekom E, van der Poel H. Impact of posterior musculofascial reconstruction on early continence after robot-assisted laparoscopic radical prostatectomy: results of a prospective parallel group trial. Eur Urol 2010;58:84-9.

5. Ficarra V, Novara G, Rosen RC, Artibani W, Carroll PR, Costello A, et al. Systematic review and meta-analysis of studies reporting urinary continence recovery after robot-assisted radical prostatectomy. Eur Urol 2012;62:405-17.

6. Burkhard FC, Kessler TM, Fleischmann A, Thalmann GN, Schumacher M, Studer UE. Nerve sparing open radical retropubic prostatectomy: does it have an impact on urinary continence? J Urol 2006;176:189-95.

7. Nandipati KC, Raina R, Agarwal A, Zippe CD. Nerve-sparing surgery significantly affects long-term continence after radical prostatectomy. Urology 2007;70:1127-30.

8. Kundu SD, Roehl KA, Eggener SE, Antenor JA, Han M, Catalona WJ. Potency, continence and complications in 3,477 consecutive radical retropubic prostatectomies. J Urol 2004;172(6 Pt 1):222731.

9. Tzou DT, Dalkin BL, Christopher BA, Cui H. The failure of a nerve sparing template to improve urinary continence after radical prostatectomy: attention to study design. Urol Oncol 2009;27:358-62.

10. Choi IY, Park S, Park B, Chung BH, Kim CS, Lee HM, et al. Development of prostate cancer research database with the clinical data warehouse technology for direct linkage with electronic medical record system. Prostate Int 2013;1:59-64.

11. Mattei A, Naspro R, Annino F, Burke D, Guida R Jr, Gaston R. Tension and energy-free robotic-assisted laparoscopic radical prostatectomy with interfascial dissection of the neurovascular bundles. Eur Urol 2007;52:687-94.

12. Lee SW, Han DH, Lee KS, Jeon SS. Effect of continuous urethro- vesical anastomosis technique in incontinence after radical retropubic prostatectomy, 1:1 matching study. Int Neurourol J 2015;19: 113-9.

13. Stember DS, Nelson CJ, Mulhall JP. Preoperative erectile function is an independent predictor for decision to spare cavernous nerves during radical prostatectomy. J Sex Med 2013;10:2101-7.

14. Lavery HJ, Prall DN, Abaza R. Active patient decision making regarding nerve sparing during radical prostatectomy: a novel approach. J Urol 2011;186:487-92.

15. Suardi N, Moschini M, Gallina A, Gandaglia G, Abdollah F, Capitanio U, et al. Nerve-sparing approach during radical prostatectomy is strongly associated with the rate of postoperative urinary continence recovery. BJU Int 2013;111:717-22.

16. Khoder WY, Waidelich R, Seitz M, Becker AJ, Buchner A, Trittschler $S$, et al. Do we need the nerve sparing radical prostatectomy techniques (intrafascial vs. interfascial) in men with erectile dysfunction? Results of a single-centre study. World J Urol 2015;33:3017.

17. Harris CR, Punnen S, Carroll PR. Men with low preoperative sexual function may benefit from nerve sparing radical prostatectomy. J Urol 2013;190:981-6.

18. Reeves F, Preece P, Kapoor J, Everaerts W, Murphy DG, Corcoran $\mathrm{NM}$, et al. Preservation of the neurovascular bundles is associated with improved time to continence after radical prostatectomy but not long-term continence rates: results of a systematic review and meta-analysis. Eur Urol 2015;68:692-704.

19. John H, Suter S, Hauri D. Effect of radical prostatectomy on urethral blood flow. Urology 2002;59:566-9.

20. John H, Sullivan MP, Bangerter U, Hauri D, Yalla SV. Effect of radical prostatectomy on sensory threshold and pressure transmission. J Urol 2000;163:1761-6.

21. Catarin MV, Manzano GM, Nobrega JA, Almeida FG, Srougi M, Bruschini $\mathrm{H}$. The role of membranous urethral afferent autonomic innervation in the continence mechanism after nerve sparing radical prostatectomy: a clinical and prospective study. J Urol 2008;180:252731. 